\title{
Plasma Levels of IL-23 and IL-17 before and after Antidepressant Treatment in Patients with Major Depressive Disorder
}

\author{
Jae-Won Kim¹, Yong-Ku Kim¹ ${ }^{\bowtie}$, Jung-A Hwang1, Ho-Kyoung Yoon¹, Young-Hoon Ko', \\ Changsu Han', Heon-Jeong Lee ${ }^{2}$, Byung-Joo Ham² and Hong Seock Lee ${ }^{3}$ \\ 1'Department of Psychiatry, Korea University, College of Medicine, Ansan Hospital, Ansan, Republic of Korea \\ ${ }^{2}$ Department of Psychiatry, Korea University, College of Medicine, Anam Hospital, Seoul, Republic of Korea \\ ${ }^{3}$ Department of Psychiatry, Kangnam Sacred Heart Hospital, Hallym University Medical Center, Seoul, Republic of Korea
}

\begin{abstract}
Objective Cytokines are believed to have a role in the pathophysiology of major depression. The alteration in levels of pro-inflammatory cytokines [interleukin $1 \beta$ (IL-1 $\beta$ ), IL-2, IL-6, IL-12, interferon $\gamma$, and tumor necrosis factor $\alpha$ ] in major depression supports the cytokine hypothesis of this illness. IL-23 and IL-17 are also pro-inflammatory cytokines, but few studies have focused on their role in major depression. This study investigated the potential role of the IL-23 and IL-17 axis in major depression.

Methods Plasma IL-23 and IL-17 levels were measured in 26 major depressive disorder (MDD) patients before and after 6-week treatment with antidepressants; these levels were measured in 28 age- and sex-matched normal controls. Depression severity was assessed using the Hamilton Depression Rating Scale (HDRS). IL-23 and IL-17 plasma levels were estimated using quantitative enzyme-linked immunosorbent assay.

Results Pre-treatment plasma levels of IL-23 and IL-17 in MDD patients were not significantly different from those of normal controls. In MDD patients, IL-23 and IL-17 levels after 6 weeks of antidepressant treatment were not different from the baseline levels. There was no significant correlation between changes in the cytokine levels and changes in the HDRS scores representing the severity of depression.

Conclusion The present study does not support a potential involvement of IL-23 and IL-17 axis in major depression. Replication and extension using a larger sample are required.

Psychiatry Investig 2013;10:294-299
\end{abstract}

Key Words Cytokine, Th17 cell, IL-23, IL-17, Immune, Major depressive disorder.

\section{INTRODUCTION}

Accumulating evidence suggests that dysregulation of immune system, particularly the cytokine system, is associated with the pathophysiology of major depressive disorder (MDD) ${ }^{1-3}$ Although the central nervous system affects the immune system via the autonomic nervous system and neuroendocrine system, the immune system inversely affects the central nervous system, via a cytokine network secreted by immune cells, to control behaviors and emotions. ${ }^{4}$ Cytokines

Received: November 10, 2012 Revised: April 18, 2013

Accepted: April 21, 2013 Available online: September 16, 2013

$\triangle$ Correspondence: Yong-Ku Kim, MD, PhD

Department of Psychiatry, Korea University Medical Center Ansan Hospital, 123 Jeokgeum-ro, Danwon-gu, Ansan 425-021, Korea

Tel: +82-31-412-5138, Fax: +82-31-412-5132, E-mail: yongku@korea.ac.kr

(a) This is an Open Access article distributed under the terms of the Creative Commons Attribution Non-Commercial License (http://creativecommons.org/licenses/bync/3.0) which permits unrestricted non-commercial use, distribution, and reproduction in any medium, provided the original work is properly cited. are both immunoregulators and modulators of neural functions. Pro-inflammatory cytokines [including interleukin 1 (IL-1), IL-6, tumor necrosis factor $\alpha$ (TNF- $\alpha$ ), and interferon $\gamma($ IFN- $\gamma)]$ play important roles in the central nervous system, such as controlling neuronal and glial activation, proliferation, differentiation, as well as affecting neuronal plasticity, synaptogenesis, and tissue repair. ${ }^{4}$

Several studies suggest that pro-inflammatory cytokine overexpression in the brain is an important factor in MDD. ${ }^{5}$ Some studies indicate that levels of monocytic pro-inflammatory cytokines (IL-6 and TNF- $\alpha$ ) are significantly higher in MDD patients and associated with immunological dysregulation in MDD. ${ }^{67}$ Moreover, the T-helper 1 (Th1; IL-2 and IFN$\gamma)$ and Th2 (IL-4) cytokine imbalance seems to play crucial roles in MDD pathogenesis. ${ }^{8}$

IL-17 is one of the Th17 cell-secreted cytokines, which include IL-17A, IL-17F, IL-6, IL-22, and granulocyte macrophage colony-stimulating factor (GM-CSF), whereas IL-23, 
along with transforming growth factor $\beta$ (TGF- $\beta$ ), is involved in the differentiation of naive CD4+ T cells into Th17 cells in a pro-inflammatory context. ${ }^{9}$ In the presence of IL-23 and TGF- $\beta$, naïve CD4+ T-cells become IL-17-producing Th17 cells via the transcription factor retinoic acid receptor-related orphan receptor $\gamma$ t. IL-17 activates fibroblasts to produce proinflammatory cytokines such as IL- 6 and TNF- $\alpha$, resulting in inflammation and tissue destruction in various autoimmune disorders. ${ }^{10}$ Thus, both IL-23 and IL-17 form an axis through Th17 cells, playing a key role in immune activation and immunopathogenesis of a number of diseases. ${ }^{11}$ There have been many studies suggesting that Th17 cells have a crucial role in several inflammatory responses and have a close association with autoimmune diseases. The IL-23/IL-17 axis is associated with several inflammatory conditions such as inflammatory bowel disease (IBD) ${ }^{12}$ psoriatic arthritis, ${ }^{13}$ multiple sclerosis, ${ }^{14}$ autoimmune myocarditis, ${ }^{15}$ and rheumatoid arthritis. ${ }^{16}$ There is some evidence that IL-17 is the major regulator of central nervous system autoimmunity. ${ }^{17}$

There have been reports that patients with psoriatic disorder frequently have psychiatric co-morbidity, particularly major depression, and biological factors may also explain the association between psoriasis and MDD. ${ }^{18}$ Patients with IBDs, such as ulcerative colitis and Crohn's disease, frequently have co-morbid depression and related disorders. ${ }^{19}$ Clinically, significant depression can occur in up to half of all patients with multiple sclerosis and is associated with increased morbidity and mortality. Considering the above findings, we hypothesize that psychopathological factors, as well as biological factors, may explain the association between these autoimmune diseases and MDD.

However, to date, compared to other monocytic, Th1, and Th2 cytokines, the IL-23 and IL-17 axis has been less investigated in the context of mood disorders. We hypothesized that Th17-related pro-inflammatory cytokines have a role in MDD pathogenesis. Our study investigated whether the plasma levels of IL-17 and IL-23 are higher in MDD patients than in normal controls. Further, we investigated the plasma levels of IL-17 and IL-23 after 6 weeks of antidepressant treatment, comparing these with baseline levels in MDD patients.

\section{METHODS}

\section{Subjects}

Twenty-six MDD patients among the psychiatric patients who were admitted to Korea University Ansan Hospital from May 2007 to December 2010 were recruited. Patients were diagnosed based on the Diagnostic and Statistical Manual of Mental Disorder (DSM-IV) for MDD.$^{20}$ Patients with co-morbid psychiatric disorders such as bipolar disorder or schizoaf- fective disorder were excluded. Patients with medical conditions (such as autoimmune or infective disorders) or receiving medications (such as corticosteroids and antibiotics) that could affect plasma cytokine levels were also excluded.

Psychiatric interviews were conducted by a trained physician using the DSM-IV Structured Clinical Interview. Depressive symptoms were assessed using the Hamilton Depression Rating Scale (HDRS) ${ }^{21}$ at baseline and 6 weeks after treatment. The baseline HDRS scores of MDD patients were $\geq 18$ (27.9 \pm 5.1).

MDD patients were treated with various antidepressants. Of the 26 patients, 7 were treated with venlafaxine (mean, $112.5 \mathrm{mg}$; range, $37.5-150 \mathrm{mg}$ ), 6 with paroxetine (mean, $27.08 \mathrm{mg}$; range, $25-37.5 \mathrm{mg}$ ), 5 with escitalopram (mean, 17 mg; range, 10-20 mg), 3 with mirtazapine (mean, $20 \mathrm{mg}$; range, $15-30 \mathrm{mg}$ ), 3 with bupropion (300 $\mathrm{mg}$ ), and 2 with fluoxetine (20 mg). The mean and standard deviation (SD) of HDRS scores after 6 weeks of antidepressant treatments in the MDD patients was $9.8 \pm 8.3$.

Twenty-eight healthy, non-psychiatric normal controls were recruited through local advertisements and received a monetary reward for their participation. They agreed to participate in the present study and were given a non-structured clinical interview. Subjects who had past psychiatric history, a family history of mental illness among first and second-degree relatives, substance abuse history, autoimmune disease, or infective disorder were excluded. All normal controls had been free from physical illness within the 2 weeks before the study. They showed normal findings from several laboratory tests, including blood chemistry, liver/thyroid function test, chest X-ray radiography, and electrocardiography. Demographic data of patients and normal controls are summarized in Table 1.

Informed consent was obtained from both the groups. This study was approved by the Korea University Ansan Hospital Institutional Review Board.

\section{Sample collection and measurement of plasma levels of IL-17 and IL-23}

Ulnar venous blood samples $(2 \mathrm{~mL})$ were collected in a lithium heparin vacuum tube for $1 \mathrm{~h}$ (from 8:00 A.M. to 9:00 A.M.) for both the groups. After centrifugation at $3000 \mathrm{rpm}$ for $10 \mathrm{~min}$, plasma samples were divided into microcentrifuge tubes (Eppendorf), and frozen immediately at $-70^{\circ} \mathrm{C}$ until use.

Plasma levels of IL-17 and IL-23 were determined by a solid-phase sandwich enzyme immunoassay using a human IL17 and IL-23 enzyme-linked immunosorbent assay kit (Wuhan EIAab Science, USCNLIFE, CHINA, cat\# E0063h and E0384h). Assays were performed in duplicate according to the manufacturer's recommendations. 
Briefly, $100 \mu \mathrm{L}$ of standards and samples were pipetted into each well and incubated at $37^{\circ} \mathrm{C}$ for $2 \mathrm{~h}$. Cytokines were bound by the detection antibody $(100 \mu \mathrm{L}$ of the detection reagents $\mathrm{A}$ and $\mathrm{B}$ ) and incubated at room temperature for $2 \mathrm{~h}$. Following a final washing procedure, $90 \mu \mathrm{L}$ of substrate solution was added to each well and incubated at $37^{\circ} \mathrm{C}$ for $30 \mathrm{~min}$. Subsequently, $50 \mu \mathrm{L}$ of stop solution was added to each well. The optical density of the color reaction was read using a microplate reader at a wavelength of $450 \mathrm{~nm}$. The intra- and inter-assay coefficients of variation for all analyses were less than $8 \%$.

\section{Statistical analyses}

We used the Statistical Package for the Social Sciences (SPSS) version 12.0 for all analyses. Regarding the relatively small sample size, we analyzed our samples by nonparametric method. Mann-Whitney test were used for comparing baseline plasma levels of IL-17, IL-23 of the both groups while Wilcoxon signed rank test examined the changes in cytokine level after antidepressant treatment. Chi-square tests were con-

Table 1. Demographic data

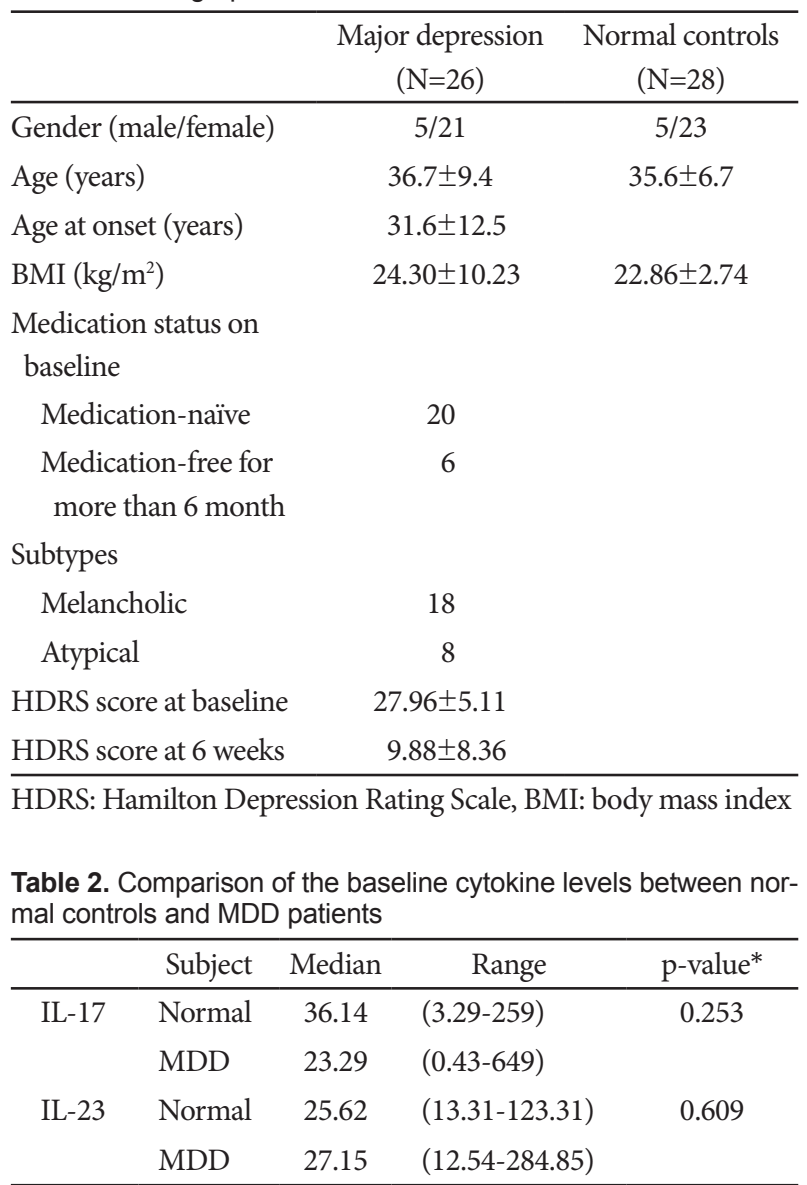

*Mann-Whitney test, $\mathrm{p}<0.05 ; \mathrm{pg} / \mathrm{mL}$. IL: interleukin, MDD: major depressive disorder ducted to analyze the association between the groups for discrete covariates. Correlations between levels of IL-17 and IL23 and HDRS score were evaluated using the Spearman's correlation test. The null hypothesis was rejected at $\mathrm{p}<0.05$.

\section{RESULTS}

\section{Demographic data}

The characteristics of the study population are listed in Table 1 . There were no significant differences in the male/female ratio $\left(\chi^{2}=0.017, \mathrm{p}=0.897\right)$, age $(\mathrm{Z}=-0.172, \mathrm{p}=0.864)$, and body mass index (BMI) $(\mathrm{Z}=-0.912, \mathrm{p}=0.362)$ between the MDD patients and normal controls.

\section{Baseline plasma cytokine levels in MDD patients and normal controls}

IL-17 and IL-23 was detected in all MDD patients and controls. The baseline plasma levels of IL-17 and IL-23 of MDD patients was not statistically different from those of controls (Table 2, Figure 1).

Further, no statistically significant differences were observed in cytokine levels between the melancholic and atypi-
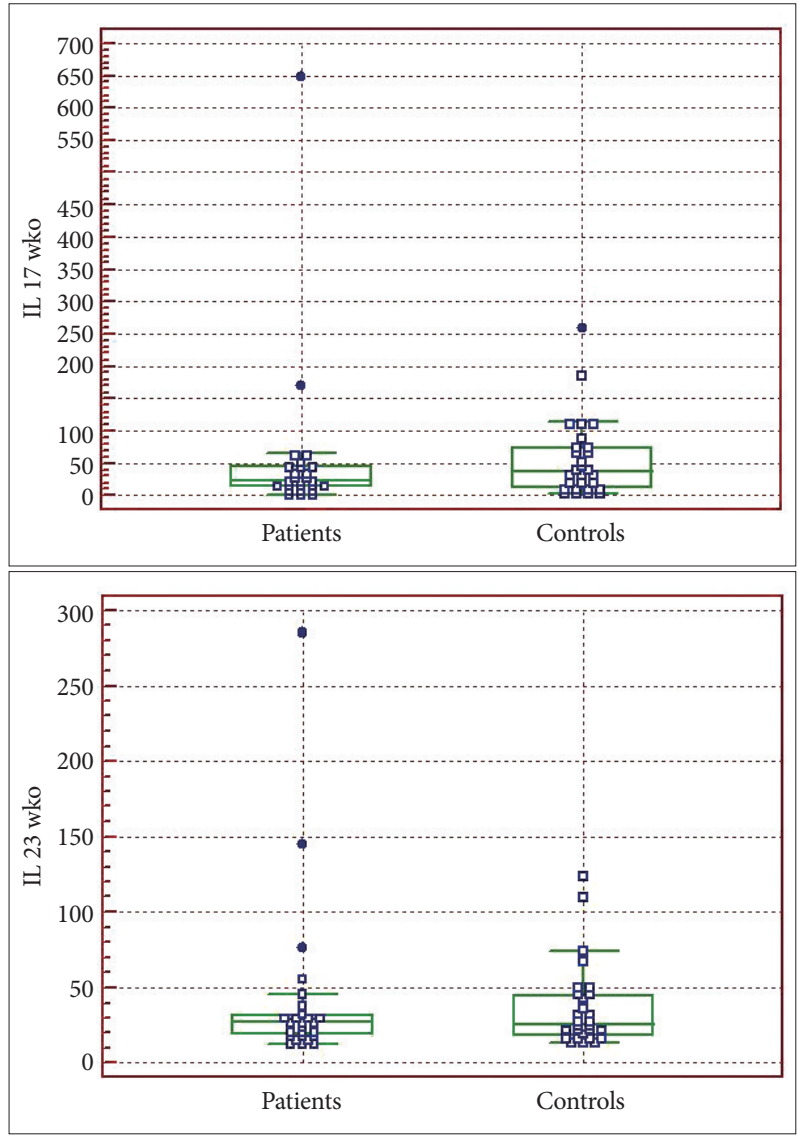

Figure 1. Comparison of the baseline cytokine levels between normal controls and MDD patients. MDD: major depressive disorder. 
cal subtypes of MDD patients ( $\mathrm{p}=0.657$ for IL-17; $\mathrm{p}=0.824$ for IL-23).

Levels of these cytokines and HDRS score of MDD patients were not significantly correlated $\left(\mathrm{r}_{\mathrm{s}}=-0.133, \mathrm{p}=0.517\right.$ for IL-17; $r_{s}=0.111, p=0.590$ for IL-23).

\section{Changes in plasma cytokine levels after antidepressant treatment in MDD patients}

In MDD patients, IL-17 and IL-23 levels did not significantly change after 6 weeks of antidepressant treatments (Table 3, Figure 2). The changes in cytokine levels after 6 weeks of antidepressant treatment and the HDRS score at 6 weeks

Table 3. Changes in plasma cytokine levels in MDD patients after antidepressant treatment

\begin{tabular}{ccclc}
\hline & Week & Median & \multicolumn{1}{c}{ Range } & p-value* \\
\hline IL-17 & 0 & 23.29 & $(0.43-649)$ & 0.830 \\
& 6 & 29 & $(6.14-224.71)$ & \\
IL-23 & 0 & 27.15 & $(12.54-284.85)$ & 0.914 \\
& 6 & 25.23 & $(12.54-527.92)$ & \\
\hline
\end{tabular}

*Wilcoxon signed rank test, $\mathrm{p}<0.05$; pg/mL. IL: interleukin, MDD: major depressive disorder

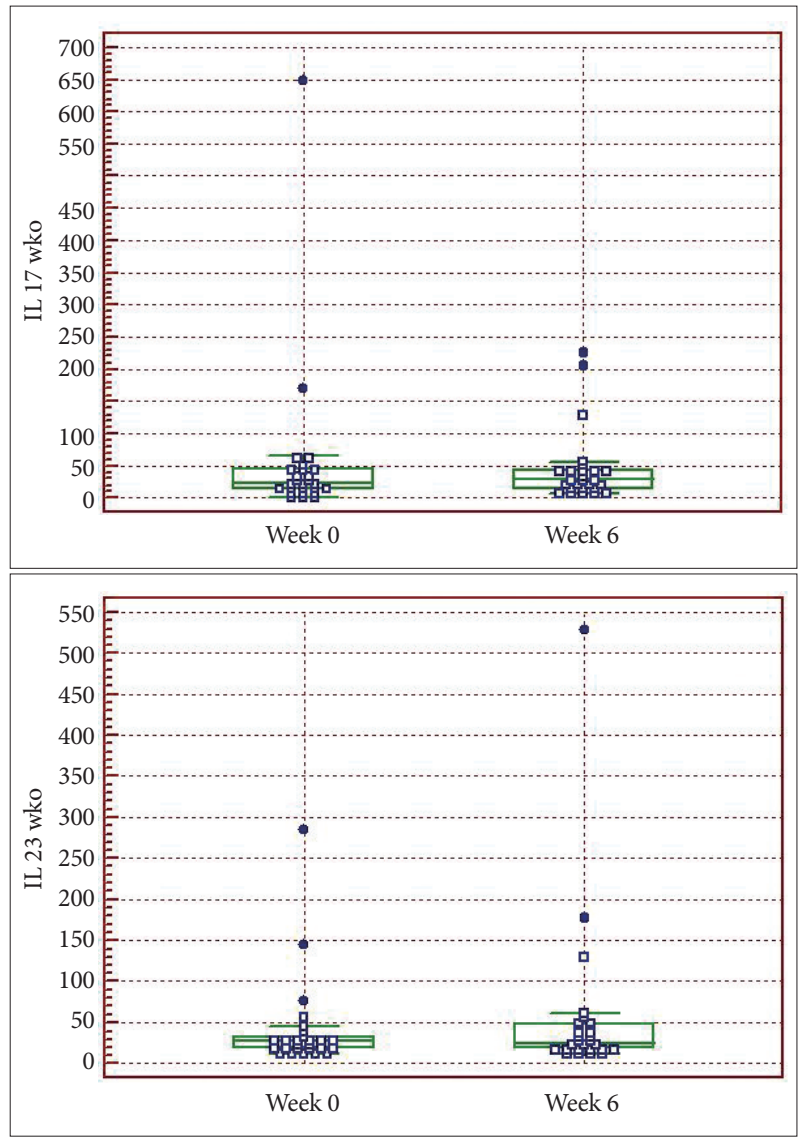

Figure 2. Comparison of cytokine levels in MDD patients at baseline and 6 weeks after antidepressant treatment. MDD: major depressive disorder. after treatment were not significantly correlated $\left(\mathrm{r}_{\mathrm{s}}=0.113\right.$, $\mathrm{p}=0.581$ for IL-17; $\mathrm{r}_{\mathrm{s}}=-0.099, \mathrm{p}=0.629$ for IL-23).

\section{DISCUSSION}

The first major finding of our study is that IL-23 and IL-17 levels in MDD patients were not significantly higher than those in normal controls. Another study reported that increased IL-17 levels are associated with the severity of anxiety in rheumatoid arthritis patients. ${ }^{22}$ These studies indicate a possibility that IL-17, among the cytokines related to Th17 cells, may be associated with anxiety symptoms in autoimmune disease, not with psychiatric diseases. However, Chen et al. reported that a Th17/Treg imbalance exists in MDD patients, which may have implications for the pathogenic role of Th17 cells in MDD. ${ }^{23}$ There is difference between Chen's study and our study; Chen's study showed relative male preference in gender distribution but our study included relatively more female subjects.

The second major finding of this study is that IL-23 and IL17 levels in MDD patients did not decreased after 6 weeks of antidepressant treatment. A previous study reported various antidepressants, including tricyclic antidepressants, selective serotonin re-uptake inhibitors (SSRIs), and serotonin-noradrenaline reuptake inhibitors, show negative immunoregulatory effects by suppressing the IFN-/IL-10 production ratio. ${ }^{24} \mathrm{Mo}-$ reover, many studies reported decreased levels of various proinflammatory cytokines after antidepressant treatment. ${ }^{25-28}$ However, our findings does not support a potential negative immunoregulatory effects of IL-23 and IL-17 axis after antidepressants treatment.

Recently, the cytokine hypothesis of depression has received attention for several reasons. First, chronic inflammatory diseases are frequently associated with depression. Second, the association between immune dysfunction and MDD is bidirectional. Not only are serum levels of many pro-inflammatory cytokines increased in depressive disorder, but also the medications that affect the cytokine system affect mood. Endotoxin administration in humans results in sickness behavior, whereas endotoxin has little effects on physical sickness symptoms. ${ }^{29}$ Third, these sickness behaviors improve when treated with antidepressants. ${ }^{30}$ Postmortem studies of brain tissues from drug-naïve MDD patients have shown increased expression of pro-inflammatory cytokines. ${ }^{31}$

Although the majority of studies support the cytokine hypothesis of MDD which implies positive association between immune dysregulation and MDD, there also have been some reports against this theory. There have been studies of outpatients with depressive disorder that showed no significant differences in pro-inflammatory cytokine profiles when com- 
pared to normal controls. ${ }^{32-34}$ If immune dysregulation causes elevation of cytokine levels in MDD, remission or even a reduction in depressive symptoms would be expected to reduce the cytokine levels. However, Hannestad et al. reported that a meta-analysis of various studies failed to show a statistically significant effect of antidepressant treatment on serum levels of IL- 6 and TNF- $\alpha .{ }^{35}$ Another group of studies reported that SSRI treatment did not significantly change TNF- $\alpha$ serum levels regardless of the reduction in depressive symptoms. ${ }^{36,37}$ Our results conflict the cytokine theory of major depression. In our study, the HDRS score significantly reduced after 6 weeks of antidepressant treatment (27.96 \pm 5.11 to $9.88 \pm 8.36)$. However, no significant differences in cytokine levels were observed, despite the marked improvement of depressive symptoms.

Our results have some limitations. First, the relatively small sample size may have limited the power of a possible association between the IL-23/IL-17 axis and major depression. Second, although the normal controls underwent a clinical interview, subclinical depressive symptoms might not be detected. Psychological stresses may affect the cytokine profiles of normal controls, resulting in increased pro-inflammatory cytokine levels.

However, in addition to the limitations, our study has various strengths. Numerous confounding factors that can affect the plasma levels of cytokines, such as gender, age, and BMI, ${ }^{38}$ were controlled. We tried to exclude the effect of drug use. Twenty patients were medication-naïve and another 6 patients were enrolled after a medication-free period of at least 6 months. Patients with medication-free period of less than 6 months were excluded because use of antidepressants can affect serum cytokine levels. Further, we tried to minimize the difference of age and BMI between the MDD patients and control groups; no statistically significant differences were found in age and BMI. Although we collected samples from groups with a similar male/female ratio, we could not explore the effect of gender on cytokines due to the preponderance of females (5 males and 21 females in MDD and 5 males and 23 females in controls). This factor should be focused on in future studies.

In conclusion, we failed to find a statistically significant association between the IL-23/IL-17 axis and MDD. However, there have been some reports suggesting a pathogenic role of Th17 cells in MDD. Future studies with larger sample sizes are necessary to confirm our results.

\section{Acknowledgments} J-W.

This paper was done as a part of master's thesis (in Medicine) of Dr. Kim

\section{REFERENCES}

1. Dubas-Slemp H, Marmurowska-Michalowska H, Szuster-Ciesielska A, Kaminska T, Kandefer-Szerszen M. The role of cytokines in depression. Psychiatr Pol 2003;37:787-798.

2. Schiepers OJ, Wichers MC, Maes M. Cytokines and major depression. Prog Neuropsychopharmacol Biol Psychiatry 2005;29:201-217.

3. Irwin MR, Miller AH. Depressive disorders and immunity: 20 years of progress and discovery. Brain Behav Immun 2007;21:374-383.

4. Munoz-Fernandez MA, Fresno M. The role of tumour necrosis factor, interleukin 6, interferon-gamma and inducible nitric oxide synthase in the development and pathology of the nervous system. Prog Neurobiol 1998;56:307-340.

5. Szelenyi J. Cytokines and the central nervous system. Brain Res Bull 2001;54:329-338.

6. Kim YK, Na KS, Shin KH, Jung HY, Choi SH, Kim JB. Cytokine imbalance in the pathophysiology of major depressive disorder. Prog Neuropsychopharmacol Biol Psychiatry 2007;31:1044-1053.

7. Dowlati Y, Herrmann N, Swardfager W, Liu H, Sham L, Reim EK, et al. A meta-analysis of cytokines in major depression. Biol Psychiatry 2010; 67:446-457.

8. Myint AM, Leonard BE, Steinbusch HW, Kim YK. Th1, Th2, and Th3 cytokine alterations in major depression. J Affect Disord 2005;88:167173.

9. Duvallet E, Semerano L, Assier E, Falgarone G, Boissier MC. Interleukin-23: a key cytokine in inflammatory diseases. Ann Med 2011;43: 503-511.

10. Vojdani A, Lambert J. The Role of Th17 in Neuroimmune Disorders: Target for CAM Therapy. Part I. Evid Based Complement Alternat Med 2011;2011:927294.

11. Tang C, Chen S, Qian H, Huang W. Interleukin-23: as a drug target for autoimmune inflammatory diseases. Immunology 2012;135:112-124.

12. Shen W, Durum SK. Synergy of IL-23 and Th17 cytokines: new light on inflammatory bowel disease. Neurochem Res 2010;35:940-946.

13. Maeda S, Hayami Y, Naniwa T, Ueda R. The Th17/IL-23 Axis and Natural Immunity in Psoriatic Arthritis. Int J Rheumatol 2012;2012:539683.

14. Hong J, Hutton GJ. Regulatory effects of interferon-beta on osteopontin interleukin-17 expression in multiple sclerosis. J Interferon Cytokine Res 2010;30:751-757.

15. Liu Y, Zhu H, Su Z, Sun C, Yin J, Yuan H, et al. IL-17 contributes to cardiac fibrosis following experimental autoimmune myocarditis by a PKCbeta/Erk1/2/NF-kappaB-dependent signaling pathway. Int Immunol 2012;24:605-612.

16. Kageyama Y, Kobayashi H, Kato N. Infliximab treatment reduces the serum levels of interleukin-23 in patients with rheumatoid arthritis. Modern Rheumatol 2009;19:657-662.

17. Stromnes IM, Cerretti LM, Liggitt D, Harris RA, Goverman JM. Differential regulation of central nervous system autoimmunity by $\mathrm{T}(\mathrm{H}) 1$ and $\mathrm{T}(\mathrm{H}) 17$ cells. Nat Med 2008;14:337-342.

18. Misery L. Depression and psoriasis. Ann Dermatol Venereol 2012;139 (Suppl 2):S53-S57.

19. Feher J, Kovacs I, Balacco Gabrieli C. [Role of gastrointestinal inflammations in the development and treatment of depression]. Orv Hetil 2011;152:1477-1485.

20. American Psychiatric Association. Diagnostic and Statistical Manual of Mental Disorders. Washington DC: American Psychiatric Press; 2000.

21. Hamilton M. A rating scale for depression. J Neurol Neurosurg Psychiatry 1960;23:56-62.

22. Liu Y, Ho RC, Mak A. The role of interleukin (IL)-17 in anxiety and depression of patients with rheumatoid arthritis. Int J Rheum Dis 2012; 15:183-187.

23. Chen Y, Jiang T, Chen P, Ouyang J, Xu G, Zeng Z, et al. Emerging tendency towards autoimmune process in major depressive patients: $\mathrm{A}$ novel insight from Th17 cells. Psychiatry Res 2011;188:224-230.

24. Kubera M, Lin AH, Kenis G, Bosmans E, van Bockstaele D, Maes M. 
Anti-Inflammatory effects of antidepressants through suppression of the interferon-gamma/interleukin-10 production ratio. J Clin Psychopharmacol 2001;21:199-206.

25. Sluzewska A, Rybakowski JK, Laciak M, Mackiewicz A, Sobieska M, Wiktorowicz K. Interleukin-6 serum levels in depressed patients before and after treatment with fluoxetine. Ann N Y Acad Sci 1995;762:474476.

26. Seidel A, Arolt V, Hunstiger M, Rink L, Behnisch A, Kirchner H. Cytokine production and serum proteins in depression. Scand J Immunol 1995;41:534-538.

27. O’Brien SM, Scully P, Fitzgerald P, Scott LV, Dinan TG. Plasma cytokine profiles in depressed patients who fail to respond to selective serotonin reuptake inhibitor therapy. J Psychiatr Res 2007;41:326-331.

28. Lee KM, Kim YK. The role of IL-12 and TGF-betal in the pathophysiology of major depressive disorder. Int Immunopharmacol 2006;6: 1298-1304.

29. Reichenberg A, Yirmiya R, Schuld A, Kraus T, Haack M, Morag A, et al. Cytokine-associated emotional and cognitive disturbances in humans. Arch Gen Psychiatry 2001;58:445-452.

30. Schramm TM, Lawford BR, Macdonald GA, Cooksley WG. Sertraline treatment of interferon-alfa-induced depressive disorder. Med J Aust 2000;173:359-361.

31. Shelton RC, Claiborne J, Sidoryk-Wegrzynowicz M, Reddy R, Aschner $\mathrm{M}$, Lewis DA, et al. Altered expression of genes involved in inflammation and apoptosis in frontal cortex in major depression. Mol Psychia- try 2011;16:751-762.

32. Brambilla F, Monteleone P, Maj M. Interleukin-1beta and tumor necrosis factor-alpha in children with major depressive disorder or dysthymia. J Affect Disord 2004;78:273-277.

33. Carpenter LL, Heninger GR, Malison RT, Tyrka AR, Price LH. Cerebrospinal fluid interleukin (IL)-6 in unipolar major depression. J Affect Disord 2004;79:285-289.

34. Marques-Deak AH, Neto FL, Dominguez WV, Solis AC, Kurcgant D, Sato F, et al. Cytokine profiles in women with different subtypes of major depressive disorder. J Psychiatr Res 2007;41:152-159.

35. Hannestad J, DellaGioia N, Bloch M. The effect of antidepressant medication treatment on serum levels of inflammatory cytokines: a metaanalysis. Neuropsychopharmacology 2011;36:2452-2459.

36. Eller T, Vasar V, Shlik J, Maron E. Pro-inflammatory cytokines and treatment response to escitalopram in major depressive disorder. Prog Neuropsychopharmacol Biol Psychiatry 2008;32:445-450.

37. Haastrup E, Knorr U, Erikstrup C, Kessing LV, Ullum H. No evidence for an anti-inflammatory effect of escitalopram intervention in healthy individuals with a family history of depression. J Neuroimmunol 2012; 243:69-72.

38. Haack M, Hinze-Selch D, Fenzel T, Kraus T, Kuhn M, Schuld A, et al. Plasma levels of cytokines and soluble cytokine receptors in psychiatric patients upon hospital admission: effects of confounding factors and diagnosis. J Psychiatr Res 1999;33:407-418. 